\title{
Remote Ischemic Conditioning Protects Diabetic Retinopathy in Streptozotocin-induced Diabetic Rats via Anti-Inflammation and Antioxidation
}

\author{
Changhong Ren ${ }^{1,5}$, Hang Wu ${ }^{1,2}$, Dongjie Li1 ${ }^{1,2}$, Yong Yang ${ }^{3}$, Yuan Gao ${ }^{1,2}$, Yunneng Jizhang ${ }^{4}$, Dachuan Liu ${ }^{2}$, \\ Xunming Ji ${ }^{1,5}$, Xuxiang Zhang ${ }^{1,2, ~ *}$ \\ ${ }^{1}$ Beijing Key Laboratory of Hypoxia Conditioning Translational Medicine, Beijing, China. \\ ${ }^{2}$ Department of Ophthalmology, Xuanwu Hospital, Capital Medical University, Beijing, China. \\ ${ }^{3}$ Department of Herbal Formula Science Medicine, Chinese Medicine College, Beijing University of Chinese \\ Medicine, Beijing, China. \\ ${ }^{4}$ Center of Cerebrovascular Disease Research, University of Pittsburgh School of Medicine, Pittsburgh, USA. \\ ${ }^{5}$ Center of Stroke, Beijing Institute for Brain Disorder, Beijing 100069, China
}

[Received May 24, 2018; Revised June 24, 2018; Accepted July 11, 2018]

\begin{abstract}
Ischemic conditioning inhibits oxidative stress and inflammatory response in diabetes. However, whether limb remote ischemic conditioning (LRIC) has beneficial effects on diabetic retinopathy (DR) remains unknown. This study aims to investigate the protective effects of LRIC in retinal ganglion cell in streptozotocin (STZ) induced Type 1 diabetic rats. A total of 48 healthy male Sprague-Dawley (200-220g) rats were randomly assigned to the normal group, normal+LRIC group, diabetes mellitus (DM) group and DM+LRIC group. Streptozotocin (STZ, $60 \mathrm{mg} / \mathrm{kg}$ ) was intraperitoneally injected into the rats to establish the diabetic model. LRIC was conducted by tightening a tourniquet around the upper thigh and releasing for three cycles daily (10 mins $x$ 3 cycles). Retinas were harvested after 12 weeks of LRIC treatment for histopathologic, Western blot and ELISA analysis. Plasma were collected at the same time for ELISA analysis. LRIC alleviated diabetic retinopathy symptoms as evidenced by the increased number of retinal ganglion cells $(P<0.01)$ and decreased glial fibrillary acidic protein (GFAP) expression level $(P<0.01)$ in the rat retina. $L R I C$ in DM rats exhibited anti-inflammatory and antioxidative effects as confirmed by the down-regulation of pro-inflammatory cytokine: interleukin-6 (IL6), and the up-regulation of antioxidants: superoxide dismutase (SOD), and glutathione (GSH)/oxidized glutathione (GSSG). Furthermore, LRIC significantly downregulated VEGF protein expression in the retina $(P<0.01)$. These results suggest that the antioxidative and anti-inflammatory activities of LRIC may be important mechanisms involved in the protective effect of LRIC in STZ-induced diabetic rats.
\end{abstract}

Key words: diabetes mellitus, diabetic retinopathy, limb remote ischemic conditioning, oxidative stress

Diabetic retinopathy (DR), a leading cause of blindness, is one of the complications associated with diabetes [1]. increasing evidence support the idea that retinal neurons undergo degeneration, which may be part of vision impairment in the early stage of diabetes. In particular, retinal ganglion cells, which are predominantly responsible for conducting visual impulses to the brain [2]. However, effective neuroprotective agents are limited in preventing retinal neurons death in DR [2]. Currently, the most effective therapeutic options such as laser photocoagulation, corticosteroids, anti-vascular endothelial growth factor (anti-VEGF) agents and

*Correspondence to: Dr. Xuxiang Zhang Xuanwu Hospital, Capital Medical University, Beijing 100053 China. E-mail: zhang_xuxiang@hotmail.com.

Copyright: ( 2018 Ren C et al. This is an open-access article distributed under the terms of the Creative Commons Attribution License, which permits unrestricted use, distribution, and reproduction in any medium, provided the original author and source are credited. 
vitrectomy, are limited due to their considerable side effects (e.g. glaucoma) [3, 4]. Therefore, developing novel, mechanism-based therapeutic strategies is highly desirable for the clinical management of DR patients [5].

Hyperglycemia-mediated oxidative stress and inflammation play important roles in the pathogenesis of DR $[3,4,[6]]$. Shifting the delicate balance between the production and elimination of oxygen radicals or inflammatory cytokines can result in cellular damage. Long-term hyperglycemia induces the overproduction of reactive oxygen species (ROS) that triggers oxidative stress. Low activities of antioxidative enzymes contribute to further accumulation of ROS. It has been reported that necrosis could cause oxidative stressinduced retinal cell death in turn promoting the expression of pro-inflammatory genes. Upregulation of proinflammatory cytokines such as TNF- $\alpha$ and interleukin-1 $\beta$ (IL-1 $\beta$ ) were observed in the eyes of patients with DR [7]. TNF- $\alpha$ also upregulated the expression of VEGF in the retina in early stages of diabetes, which was associated with increased vascular permeability and disruption of the blood-retinal barrier $[8,9]$. Thus, treatments that limit oxidative and inflammatory effects could be highly beneficial for DR patients by reducing or preventing retinal complications.

Ischemic conditioning has been considered a form of protection against ischemic injury in a variety of organs through the initiation of endogenous protective mechanisms [10, 11]. Retinal ischemic conditioning induced by increasing intraocular pressure was reported to prevent and reduce retinal function impairment in both Type 1 and Type 2 diabetic animal models [12,13]. However, there are significant limitations pertaining to local ischemic conditioning due to the characteristics and properties of inducing ischemic conditioning in vital organs such as the heart or brain. Many groups have demonstrated that limb remote ischemic conditioning (LRIC) has a neuroprotective effect in both experimental and clinical studies [14]. Brandli et al reported that LRIC had a protective effect by significantly increasing the amplitude of $a-$ and $b$ waves in a light-induced retinal damage animal model [15]. LRIC twice daily could reduce sensitive Creactive protein (CRP) and IL-6 in intracranial arterial stenosis patients [16]. Recently, we reported that repeated daily LRIC attenuated ischemia/reperfusioninduced retinal injury in rats through the increase in antioxidative defenses [17]. Taken together, the aim of the present study was to examine whether LRIC has therapeutic effects in DR prevention by modulating oxidative stress and inflammation.

\section{MATERIALS AND METHODS}

\section{Animal models}

48 healthy male Sprague-Dawley rats $(240-250$ g) were used for this study. Type $1 \mathrm{DM}$ was induced by a single injection of $1 \%$ streptozotocin (STZ, Sigma, Buchs, Switzerland) intraperitoneally $(60 \mathrm{mg} / \mathrm{kg}$ freshly dissolved in citrate buffer, $\mathrm{pH}$ 4.2). The other groups received the same volume of citrate buffer $(\mathrm{pH} 4.2)$. After three days, rats with random blood glucose $>16.7$ $\mathrm{mmol} / \mathrm{L}$ were included. Diabetic rats were randomly assigned to DM or DM+LRIC groups. Blood glucose level and weight for all rats were monitored every week.

\section{Limb remote ischemia conditioning protocol}

Remote ischemia preconditioning (LRIC) was conducted as previously described [17]. Rats were anesthetized with sodium pentobarbital $(30 \mathrm{mg} / \mathrm{kg})$ intraperitoneally. LRIC was conducted by tightening a tourniquet around the upper thigh and releasing for three cycles daily. Each cycle comprises $10 \mathrm{~min}$ of ischemia and $10 \mathrm{~min}$ of reperfusion. LRIC was applied at 4 days after STZ injection and then repeated every day thereafter for up to 12 weeks (Fig. 1A). Rats in the control and diabetic groups received sodium pentobarbital treatment alone. Anal temperature was maintained using heating pads. There were no negative effects on the function or tissue integrity of the limb [18, 19].

\section{Tissue processing}

After anesthesia, rats were sacrificed and their eyes enucleated. The left retinas were isolated and stored at $80{ }^{\circ} \mathrm{C}$. The right eyes were fixed in $4 \%$ paraformaldehyde for 4 hours at $4^{\circ} \mathrm{C}$. The cornea and lens were removed, and eye cups were dehydrated and embedded in paraffin. The tissues were sectioned at a thickness of $3 \mu \mathrm{m}$ along the horizontal meridian through the optic nerve head. Tissue sections were then stained with hematoxylin and eosin.

\section{Immunohistochemistry}

Tissue sections were deparaffinized and washed in 0.1 M sodium phosphate buffered saline (PBS) [20], then processed with $3 \% \mathrm{H}_{2} \mathrm{O}_{2}$, incubated in $1 \%$ BSA for 1 hour and incubated with primary antibodies anti-Brn3a (1:100, Santa Cruz Biotechnology, CA) and anti-glial fibrillary acidic protein (GFAP, 1:100, Santa Cruz Biotechnology, CA) at $4^{\circ} \mathrm{C}$ overnight. After washing with PBS, sections were incubated with the appropriate 
secondary antibodies for 1 hour at room temperature. The area of the retina was measured with the computer program Image Pro Plus (IPP, Media Cybernetics) in a single-blind fashion by an independent investigator. GFAP staining was quantified from three images. The number of positive cells in the ganglion cell layer was measured at $200 \mu \mathrm{m}$ away from the optic nerve head by an independent investigator. The average was then calculated and presented as the representative value for each group.

\section{Measurement of ROS production}

The method for detecting ROS was performed as described previously [21]. Homogenized retinal samples taken from the rats were diluted to $10 \mathrm{mg} / \mathrm{mL}$ after measuring protein concentration using the bicinchoninic acid (BCA) method. After 30 minutes of incubation, $\mathrm{H}_{2} \mathrm{O}_{2}$ levels in brain homogenates were determined using $50 \mu \mathrm{mol} / \mathrm{L}$ Amplex red, $0.1 \mathrm{U} / \mathrm{mL}$ horseradish peroxidase, and respiratory substrates (4 $\mathrm{mmol} / \mathrm{L}$ pyruvate, $2 \mathrm{mmol} / \mathrm{L}$ malate, $2 \mathrm{mmol} / \mathrm{L}$ glutamate, and $0.8 \mathrm{mmol} / \mathrm{L}$ complex $\mathrm{V}$ inhibitor oligomycin) at $37{ }^{\circ} \mathrm{C}$ on a DTX-880 Multimode Detector.

\section{Measurement of SOD and GSH/GSSG levels in plasma}

Rats were anesthetized and sacrificed after 12 weeks of LRIC treatment. For SOD/catalase ratio and GSH/GSSG ratio assays, the retinas were sonicated in PBS (pH 7.4) for two $30 \mathrm{sec}$ bursts. For glutathione quantification, the retinas were sonicated in 5\% 5sulfosalicylic acid. Total protein concentration was measured by the BCA Protein Assay kit reagent (Thermo Electron Corporation, USA). SOD and CAT activities were assayed using the SOD Assay Kit-WST (Dojindo Molecular Technologies, Gaithersburg, Maryland, USA) and Catalase Assay kit (Beyotime Biotechnology, Nanton, China), respectively. Enzyme activities were calculated as units/mg of protein and expressed as the relative value to normal control animals. The redox state of the whole body was determined as the ratio of GSH/GSSG using a GSH and GSSG Assay Kit (Beyotime Biotechnology, Nanton, China). The SOD/catalase ratio and GSH/GSSG ratio were expressed as ratios of their absolute values.

\section{Enzyme-linked immunosorbent assay (ELISA)}

Rats were anesthetized and sacrificed after 12 weeks of LRIC treatment. The levels of VEGF, IL-6, TNF- $\alpha$ and
IL-1 $\beta$ in rat retina and plasma were analyzed using ELISA according to the manufacturer's protocol (R\&D, USA). Fresh blood samples from the inferior vena cava were collected into a vacuum tube with sodium citrate (3.2\% $\mathrm{w} / \mathrm{v}$ ) and centrifuged at a speed of $3500 \mathrm{rpm}$ for $15 \mathrm{~min}$ at room temperature. Plasma were obtained and stored in $80^{\circ} \mathrm{C}$ until further analysis [22].

\section{Western blot}

Protein was isolated from the rat retina at 12 weeks after LRIC treatment. Protein $(20 \mu \mathrm{g})$ was electrophoresed on $12.5 \%$ sodium dodecyl sulfate polyacrylamide gels then transferred to a polyvinylidene difluoride membrane (Millipore Corporation, USA) [11]. Membranes were blocked for $1 \mathrm{~h}$ in $5 \%$ skim milk in 1xTris-buffered saline with $0.01 \%$ Tween-20 buffer and immersed overnight at $4{ }^{\circ} \mathrm{C}$ with the primary antibody against VEGF (1:1000; Abcam) [22]. GAPDH was used to verify equal protein loading. The specific reaction was visualized by the chemiluminescence substrate luminol reagent (GE Healthcare, UK). The optical density of protein was measured using Image-Pro Plus software 5.0 (Rockville, MD, USA) according to the manufacturer's instructions. The mean amount of protein expression from the normal group was assigned a value of 1 to serve as reference.

\section{Statistical analysis}

Data were expressed as mean \pm standard deviation (SD) and were calculated using SPSS version 19.0 (SPSS, Chicago, IL, USA). Differences between groups were analyzed using One-way analysis of variance (ANOVA). Post hoc multiple comparisons were performed using Fisher's LSD where appropriate. Comparisons of weight and blood glucose level across time points were analyzed by two-way repeated measures ANOVA. All analyses were performed with significance set at $\mathrm{P}<0.05$.

\section{RESULT}

\section{LRIC had no effect on body weight and blood glucose levels}

Diabetes induced a significant increase of blood glucose level and significant reduction of body weight when compared to the control group (Fig. 1B, C). Treatment with LRIC had no effect on blood glucose level and body weight (Fig. 1B, C) 
A

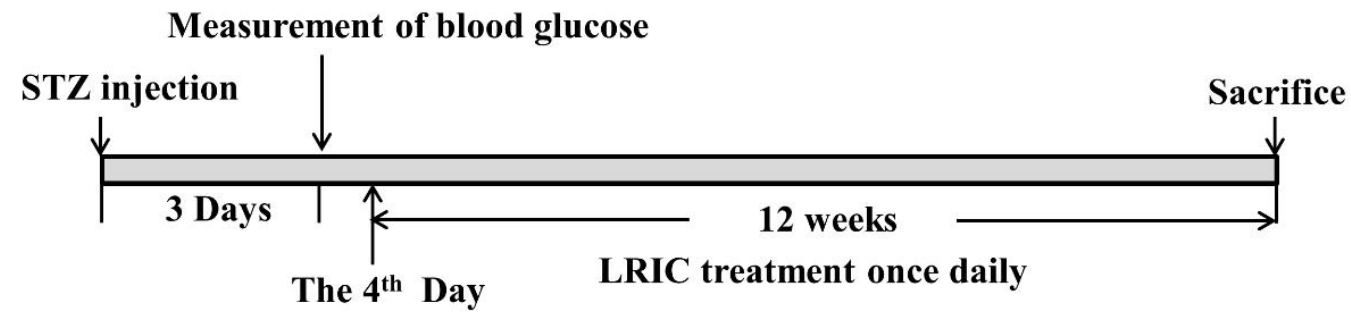

B

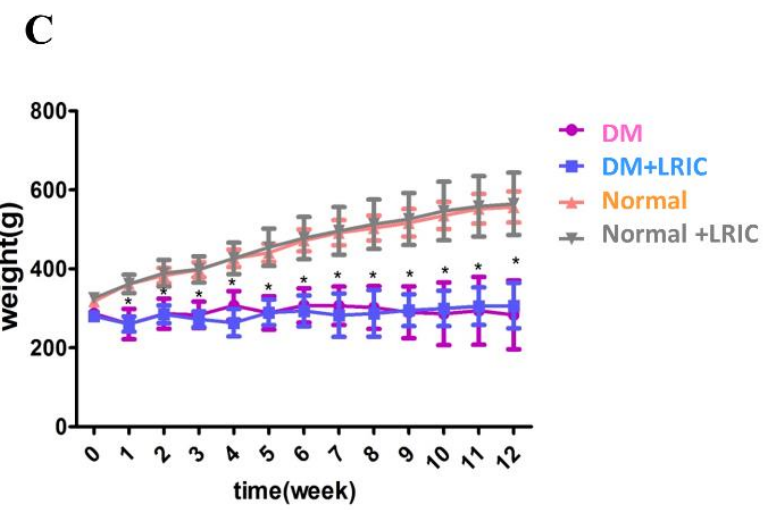

Figure 1. Effect of LRIC on blood glucose levels and body weight at 12 weeks after onset of diabetes. (A) Representative sketches of the experiment. (B) Quantification of blood glucose levels. (C) Quantification of body weight. Data are expressed as mean \pm SD, * $\mathrm{P}<0.05$ (DM vs. Normal group). $\mathrm{N}=10$ each group.

\section{LRIC treatment ameliorated Brn3a+ retinal ganglion cell loss in diabetic rats}

To demonstrate RGC loss in our diabetic rat model, we quantitatively analyzed RGCs using the Brn3a marker. Brn3a+ cells were present in the ganglion cell layer and the nuclei of cells are predominantly stained (Fig. 2). The number of Brn3a+ cells in the RGC layer in the normal group, normal+LRIC group, DM group and DM+LRIC group are $18.45 \pm 3.97,17.18 \pm 3.37$, $15.41 \pm 3.07$ and $20.18 \pm 1.64$, respectively. Compared to normal control (Fig. 2A), there was a significant decrease of Brn3a+ cells in the DM group $(\mathrm{P}<0.05$, Fig. $2 C)$. The number of RGCs was significantly increased in the DM+LRIC group (Fig. 2D) compared with the DM group $(\mathrm{P}<0.01$, Fig. $2 \mathrm{E})$. Taken together, these data indicate that LRIC could prevent Brn3a+ RGCs loss in diabetic rats.

\section{LRIC treatment ameliorated retinal Müller cell activation in diabetic rats}

To determine whether LRIC has beneficial effects on Müller cells, we also investigated the expression and localization of GFAP in rats' retinas. GFAP immunostaining was performed to evaluate glial cell activation in the diabetic retina and LRIC. In normal rats, GFAP was expressed in the inner limiting membrane and retinal nerve fiber layer (Fig. 3A). In DM rats, however, GFAP expression was significantly increased and distributed throughout the retinas (Fig. 3C). However, GFAP staining was significantly reduced in DM+LRIC rats as compared with DM rats at 12 weeks after LRIC treatment (Fig. 3D, E).

\section{LRIC treatment attenuated oxidative stress induced by hyperglycemia}

To determine whether LRIC treatment can attenuate oxidative stress induced by hyperglycemia, ROS production in the retina was evaluated at 12 weeks after LRIC treatment. In DM rats, ROS levels were significantly increased compared with normal rats $(\mathrm{P}<0.01$, Fig. 4). LRIC treatment induced a significant $(\mathrm{P}<0.01)$ reduction in ROS levels, suggesting the attenuation of oxidative damage. 

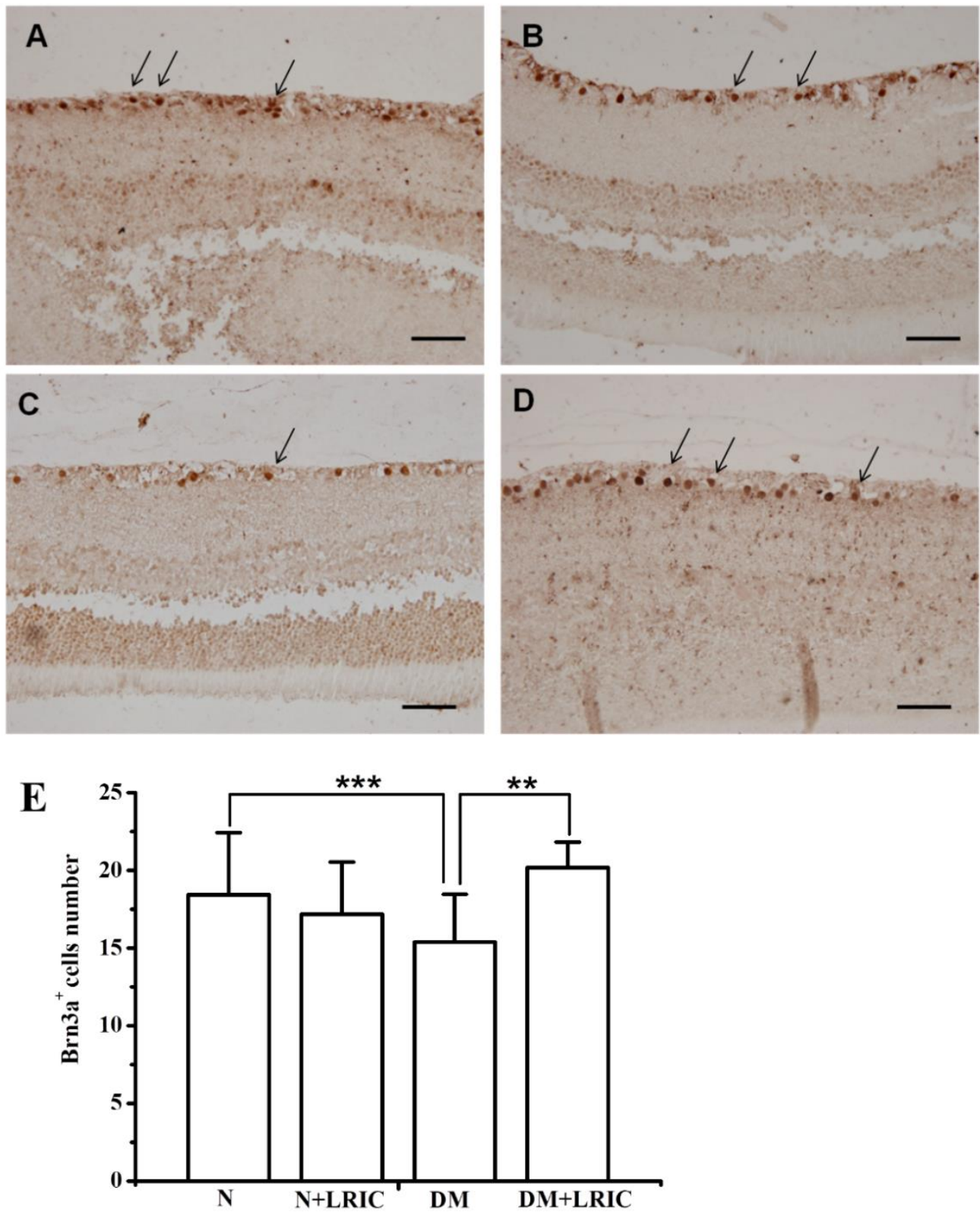

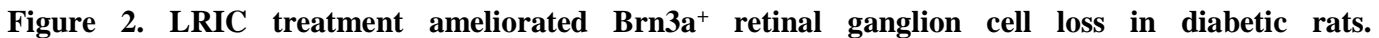
Immunohistochemical analysis of retinas of normal control group (A), normal+LRIC group (B), DM group (C) and DM+LRIC group (D). Arrows indicate Brn3a ${ }^{+}$RGCs, scale bar $=50 \mu \mathrm{m}$. (E) Bar graphs depicting the average number of $\mathrm{Brn} 3 \mathrm{a}^{+}$RGCs in each group. Data are expressed as mean $\pm \mathrm{SD}, * P<0.05$. $\mathrm{N}=5$ each group.

\section{LRIC altered antioxidant enzyme levels in diabetic rats}

SOD, catalase, and glutathione play important roles in the detoxification of ROS in the retina. To investigate the possible influence of LRIC treatment on antioxidant enzymes, we evaluated the activity of SOD, SOD/ catalase ratio, and glutathione levels at 12 weeks after LRIC treatment. Compared with normal control rats, retinal SOD and glutathione activities in DM rats were markedly decreased by $16.8 \%(\mathrm{P}<0.05)$ and $81.5 \%$ $(\mathrm{P}<0.01)$, respectively (Fig. 5A, B). LRIC treatment significantly increased SOD activity by $19.1 \%(\mathrm{P}<0.05$ versus the diabetic control group, Fig. 5). 

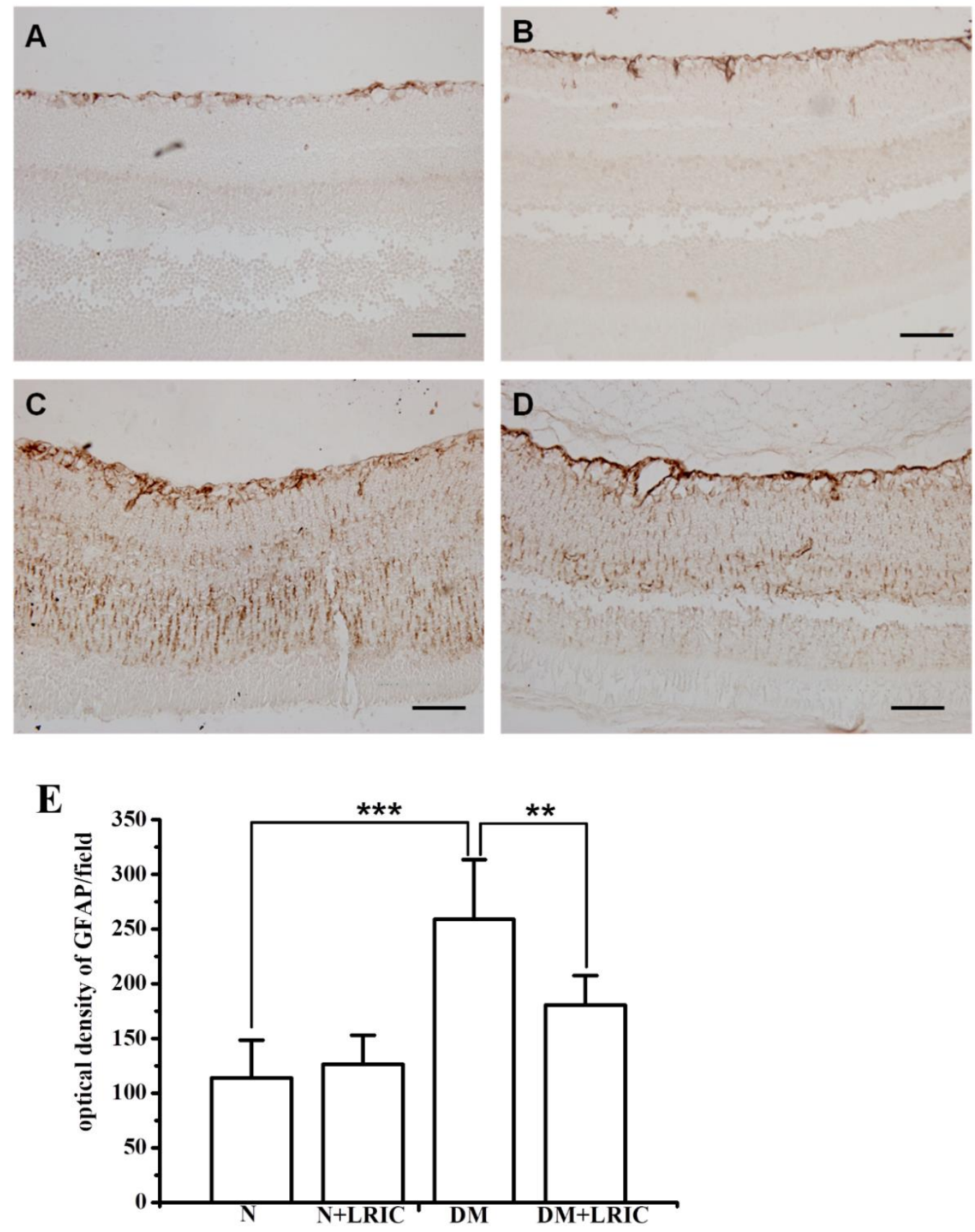

Figure 3. LRIC treatment ameliorated retinal Müller cell activation in diabetic rats. There was a significant increase in the level of GFAP expression in the diabetic retina (C) compared with the control retina (A). After 12 weeks of LRIC treatment, GFAP immunostaining decreased significantly in the diabetic retina (D). However, GFAP expression in the control retina was not affected by LRIC (B). Scale bar $=50 \mu \mathrm{m}$. (E) Bar graphs depicting the the density of GFAP in each group. Data are expressed as mean $\pm \mathrm{SD}, * * P<0.01, * * * P<0.001$. $\mathrm{N}=5$ each group.

However, LRIC had no effect on the glutathione and $\mathrm{SOD} /$ catalase ratio (Fig. 5B, C). The systemic redox status of the retina was estimated by determining the ratio of reduced glutathione (GSH)/oxidized glutathione (GSSG). The ratio of GSH/GSSG in DM rats was significantly lower compared with normal rats $(\mathrm{P}<0.001)$ (Fig. 5D). LRIC treatment significantly increased the ratio of GSH/GSSG by $19.1 \%(\mathrm{P}<0.01$ versus the diabetic control group, Fig. 5D). 


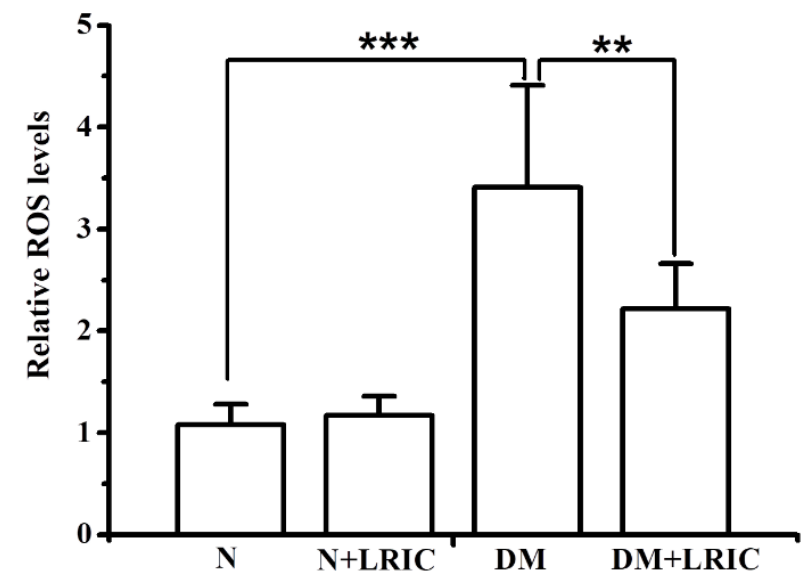

Figure 4. LRIC treatment attenuated oxidative stress induced by hyperglycemia. ROS production in the retina was evaluated at 12 weeks after LRIC treatment. The graph showed the relative reactive oxygen species levels in each group. $* P<0.05, * * P<0.01, \mathrm{~N}=7$ each group.

\section{LRIC treatment attenuated retinal inflammation in diabetic rats}

Next, we examined the effect of LRIC on inflammatory markers in the diabetic rat retina and plasma at 12 weeks after LRIC treatment. The levels of IL-6, TNF- $\alpha$ and IL$1 \beta$ in the retina were analyzed using ELISA. Compared with the normal control rat group, the levels of IL-6, TNF- $\alpha$ and IL- $1 \beta$ were markedly increased in the DM control rat group $(\mathrm{P}<0.05$, Fig. 6). LRIC treatment significantly decreased the levels of IL- 6 and TNF- $\alpha$ in the rat retina $(\mathrm{P}<0.05$, Fig. 6). LRIC treatment also significantly suppressed the level of IL-6 in the plasma (P<0.05, Fig. 6).

\section{LRIC treatment decreased the level of VEGF in diabetic rats}

Dysregulated retinal VEGF production during DR is one of the most devastating responses to oxidative stress and inflammation [22, 23]. First, we examined the VEGF protein level in plasma at 12 weeks after LRIC treatment. There was no significantly difference among the four groups (Fig. 7A). VEGF protein levels in the retina were determined using Western blotting and ELISA at 12 weeks after LRIC treatment. Compared with the normal control group, VEGF protein level was significantly increased in the retinas of DM control rats. However, LRIC treatment significantly attenuated this upregulation (Fig. 7).
$\mathbf{A}$

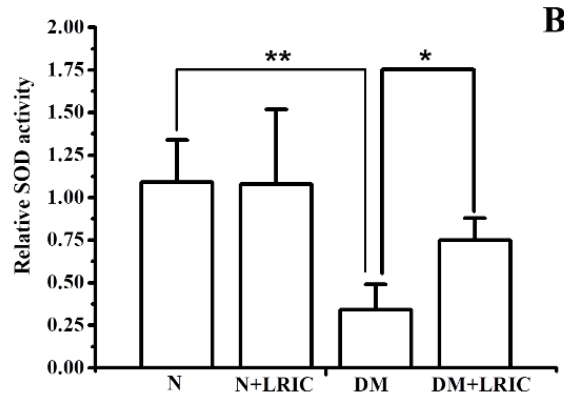

C

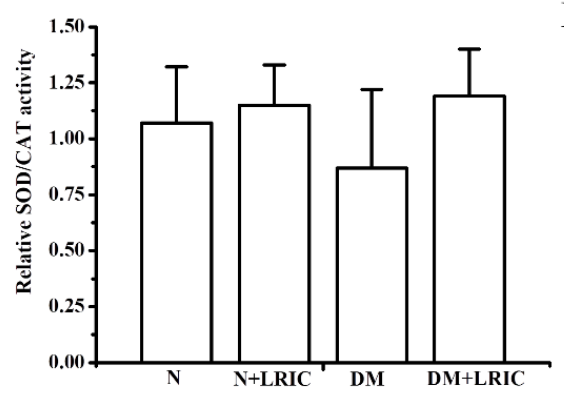

B

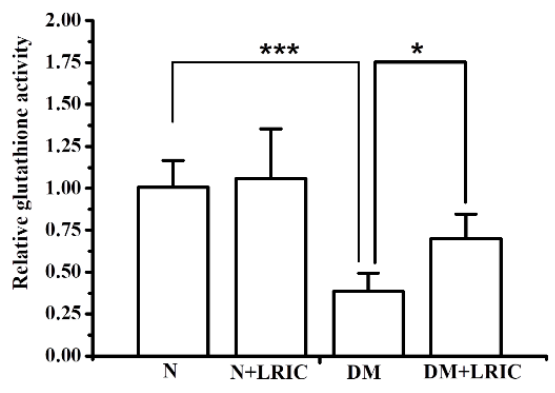

D

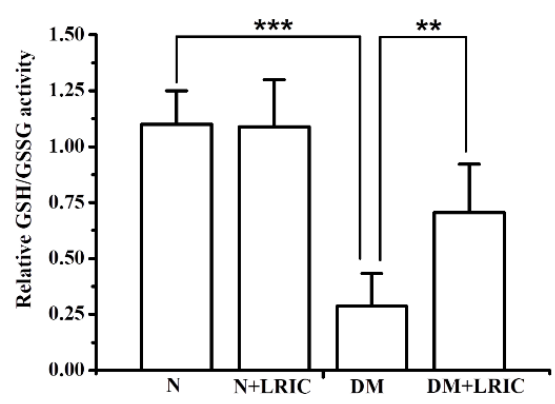

Figure 5. LRIC altered antioxidant enzyme levels in diabetic rats. Antioxidant enzyme levels in the retina were analyzed at 12 weeks after LRIC treatment. (A) Analysis of SOD activity. Data represent mean \pm SD of six independent experiments. (B) The analysis of total glutathione level. Data represent mean \pm SD of three independent experiments. (C) Analysis of SOD/CAT ratio. (D) Analysis of GSH/GSSG ratio. Data represent mean $\pm \mathrm{SD}$ of six independent experiments. $* P<0.05, * * P<0.01, \mathrm{~N}=7$ each group. 
A
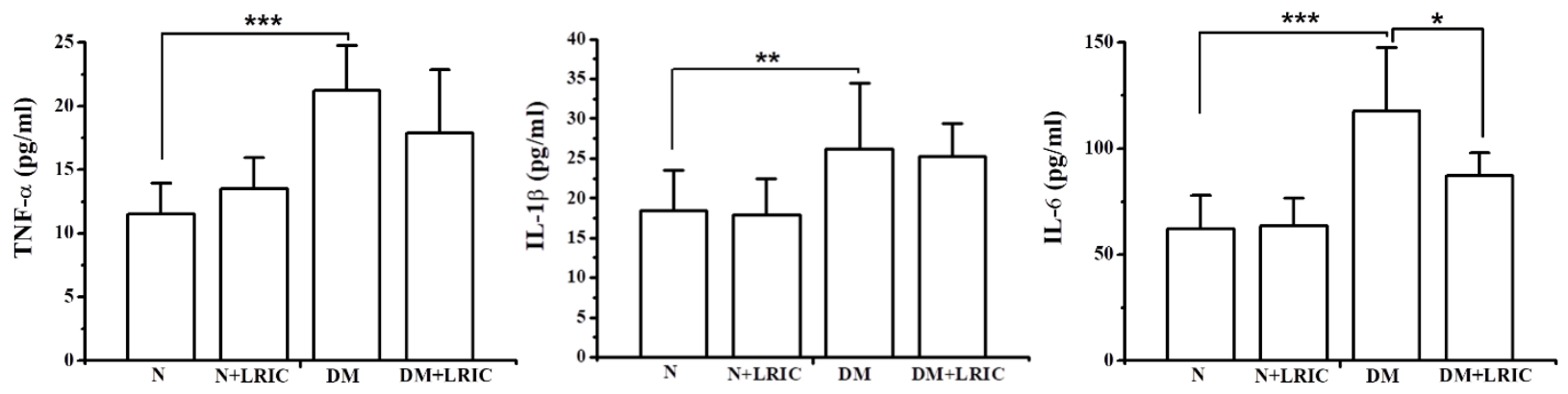

B
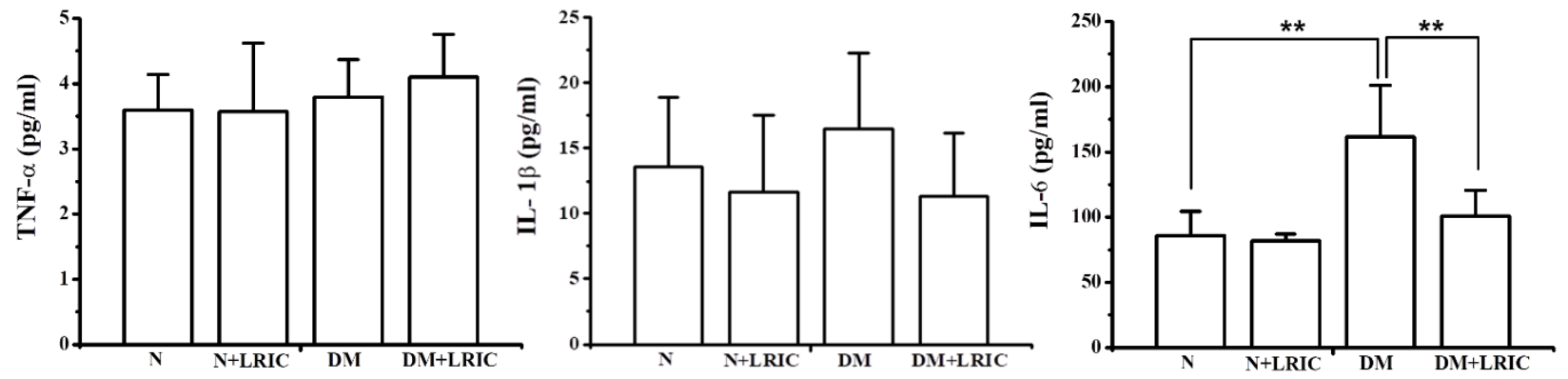

Figure 6. LRIC treatment attenuated retinal inflammation in diabetic rats. The inflammatory markers were analyzed in the diabetic rat retina and plasma at 12 weeks after LRIC treatment by using ELISA. (A) Inflammatory cytokines TNF- $\alpha$, IL-1 $\beta$ and IL-6 levels in the rat retina. (B) Inflammatory cytokines TNF- $\alpha$, IL-1 $\beta$, IL-6 levels in the rat plasma. Data represent mean \pm SD of six independent experiments. $* P<0.05, * * P<0.01, \mathrm{~N}=7$ each group.

\section{DISCUSSION}

In this study, we demonstrated that LRIC had a neuroprotective effect over diabetic retinopathy. LRIC treatment ameliorated Brn3a+ retinal ganglion cell loss and attenuated retinal Müller cell activation. LRIC reduced inflammatory cytokine (IL-6) and ROS levels as well as increase the levels of antioxidants. Taken together, this study suggests that LRIC could exert remarkable neuroprotective effects against diabetes-induced RGC degeneration in rats by attenuating the progression of diabetic retinopathy through anti-inflammatory and antioxidative mechanisms (Fig. 8).

Our results showed that LRIC had no effect on blood glucose. We speculated that the protective mechanism of LRIC on DR is not through a reduction of blood glucose level. Although diabetic patients receive intensive glycemic control (median HbA1c: 7.3\%) with conventional (median HbA1c: 9.1\%) treatment, $24 \%$ patients still develop DR [3]. We therefore suggest that LRIC be used as an adjunct therapy to delay the progression of DR.

Diabetic rodents exhibited progressive loss of RGCs in the ganglion cell layer during diabetes [14]. A reliable measure of RGC is of importance to evaluate the efficacy of RGC degenerative diseases [23]. The Brn3 family of POU-domain transcription factors plays an important role in differentiation and survival during the development of murine RGCs, thus indicating that $\mathrm{Brn} 3 \mathrm{a}$ is a specific and reliable marker for identifying RGCs [24, 25]. Using Brn3a immunohistochemical staining, we found that after 12 weeks from the onset of diabetes, the number of RGCs was reduced by approximately $17 \%$ compared with the normal control group. Our results showed that LRIC treatment attenuated the loss of RGCs. The neuroprotective effect of LRIC in the retina is consistent with our previously published observations [17]. The loss of RGC bodies is also reflected by a reduction in the number of axons in the optic nerve [26]. In a group of people with 15 years of diabetes, the thickness of the nerve fiber layer in the superior polar quadrant of the retina was significantly reduced compared with the control group [26, 27]. Although LRIC treatment had no effect in reducing blood glucose level, special attention should still be given to its role in neuroprotection. 
A

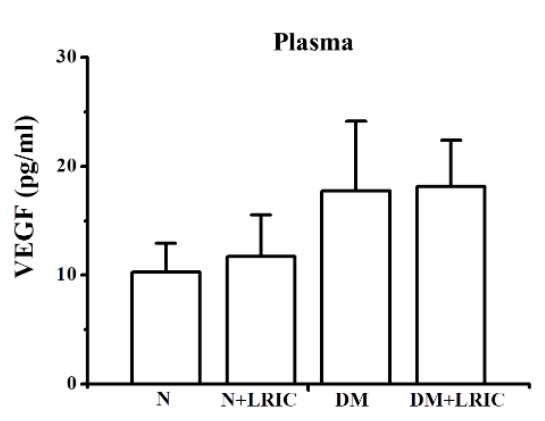

B
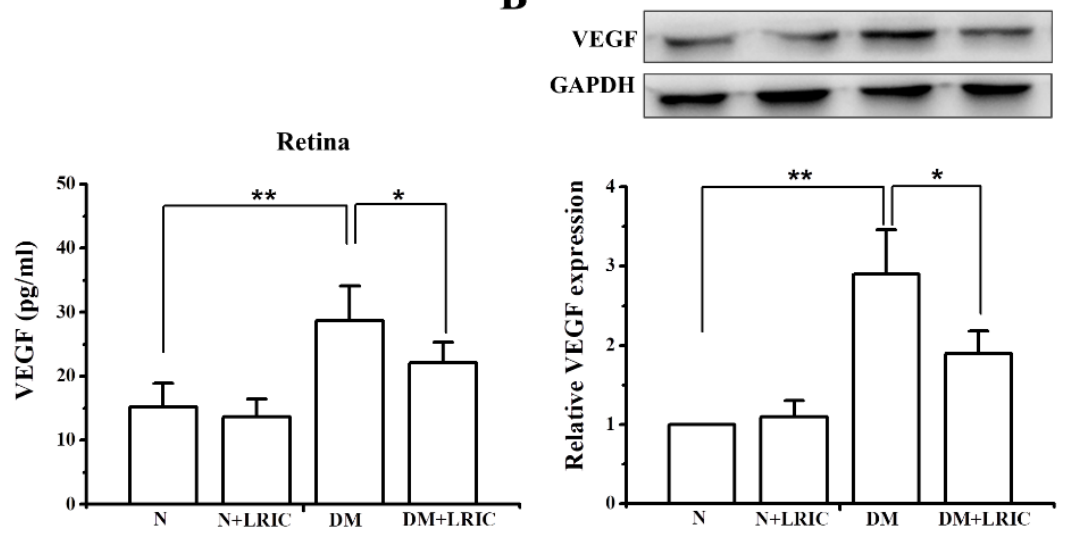

Figure 7. LRIC treatment decreased the level of VEGF in diabetic rats. The expression of VEGF was analyzed in the diabetic rat retina and plasma at 12 weeks after LRIC treatment. (A) The expression of VEGF in retinas was detected by ELISA for the different groups. (B) The expression of VEGF in retinas was detected by Western blot for the different groups. Data represent mean $\pm \mathrm{SD}$ of six independent experiments. $* \mathrm{P}<0.05, * * \mathrm{P}<0.01, \mathrm{~N}=7$ each group.

Reactive gliosis is one of the early features of the diabetic retina. Müller cells and astrocytes undergo activation in diabetes, and GFAP expression in Müller cells is widely considered to be a sensitive marker of retinal stress [28]. In the normal control retina, we found that GFAP staining was only restricted to astrocytes and not found in Müller cells. Consistent with previous studies, our results showed that GFAP is up-regulated in STZ-induced diabetic rats compared with normal control rats. After LRIC treatment, GFAP expression is decreased compared to untreated diabetic retinas, but remained higher than that in normal control retinas, indicating that LRIC inhibited Müller cell activation in diabetic rats. A similar result was observed in Fernandez's study. They showed that retinal ischemic conditioning also reduced GFAP immunoreactivity [12]. In fact, results from this study are also consistent with our previous report where LRIC reduced GFAP immunoreactivity in a retinal ischemic rat model.

It is well known that oxidative stress is an important factor in the pathology of DR. Persistent hyperglycemia induces ROS formation and triggers oxidative stress. ROS injures retinal endothelial cells by damaging proteins, lipids, and DNA [8]. In this study, we found that STZ-induced diabetic rats exhibited a higher level of ROS compared with normal control rats; LRIC treatment significantly reduced the ROS level. Excessive ROS in the retina are mainly scavenged by antioxidant enzymes such as SOD and peroxidase [29]. Our results showed that LRIC markedly increased SOD and SOD/CAT ratio as w as GSH/GSSG ratio, an index of total tissue antioxidant capacity. Our recent report showed that LRIC was able to increase SOD activity in a cerebral ischemia-reperfusion rat model [30]. Elevated inflammatory reaction also plays important roles in the pathogenesis of DR [31]. It has been reported that there were increases in the levels of pro-inflammatory cytokines including IL-1 $\beta$, IL- 6 and TNF- $\alpha$ in retinal tissues of animals and patients with DR $[32,33]$. In this study, we found that the levels of IL- $1 \beta$, IL- 6 and TNF- $\alpha$ were increased in the retinal tissue in DM only rats compared with normal control rats. LRIC treatment significantly reduced the level of IL- 6 both in the retinal tissue and plasma. This is consistent with our previous study, daily LRIC treatment reduced IL-6 plasma level in intracranial arterial stenosis patients [16]. Taken together, these observations strongly suggest that LRIC is a potential therapeutic against oxidative stress and inflammation.ell

Intraocular VEGF expression is reported to be upregulated in diabetic patients and animals [34, 35]. The increased VEGF expression triggers leukocyte aggregation, and inflammatory response [36]. This inflammation in turn stimulates a further release of VEGF $[37,38]$. Our present study showed that the expression of VEGF significantly increased in the DR rats compared with normal control rats. However, LRIC treatment significantly decreased the expression of VEGF. Contrary to our experimental results, some studies showed that ischemia or hypoxia conditioning were able to increase VEGF expression $[39,40]$. We then speculated that the reason for this discrepancy may be related to the disease model and the application method of LRIC. On the other 
hand, LRIC, an endogenous protection mechanism, could have bidirectional regulation of which remains unknown. The ischemic conditioning stimulus can be applied before ischemia (pre-conditioning), during ischemia (perconditioning), or during reperfusion after ischemia (postconditioning) [41]. Fernandez's study found that $b$ waves decreased at 6 weeks of hyperglycemia and further decreased at 10 weeks. Weekly application of $5 \mathrm{~min}$ retinal ischemia conditioning, which was induced by a transient period of brief high intraocular pressure, significantly prevented the decreased of a- and b- wave amplitude. Furthermore, delayed treatment started at 6 weeks also protected the diabetic retina from functional alteration [12] although there were no observable changes in RGCs even at 10 weeks after diabetes onset. Possible explanation for this is that functional changes may precede morphological changes in the diabetic retina. Salido's study demonstrated that retinal ischemic preconditioning was able to protect Type 2 diabetic retinopathy by reducing retinal lipid peroxidation, NOS activity, TNF $\alpha$ and VEGF levels while increasing catalase activity [13]. Research showed that axonal changes at the distal portion of the optic nerve could be the first structural change in the early stages of diabetic rats [42]. However, the retina is susceptible to ischemic injury. Compared to retinal conditioning, remote ischemic conditioning is safe, feasible, and reliable and could easily be translated to the clinic [43]. Our previous study have showed that ischemic postconditioning protected RGCs from ischemia insult at day 3 and day 7 following reperfusion, decreased GFAP expression and increased the expression of Nrf2 and HO-1 [17]. These findings in our study indicate that LRIC techniques may be a potential therapy for the treatment of DR in clinic.

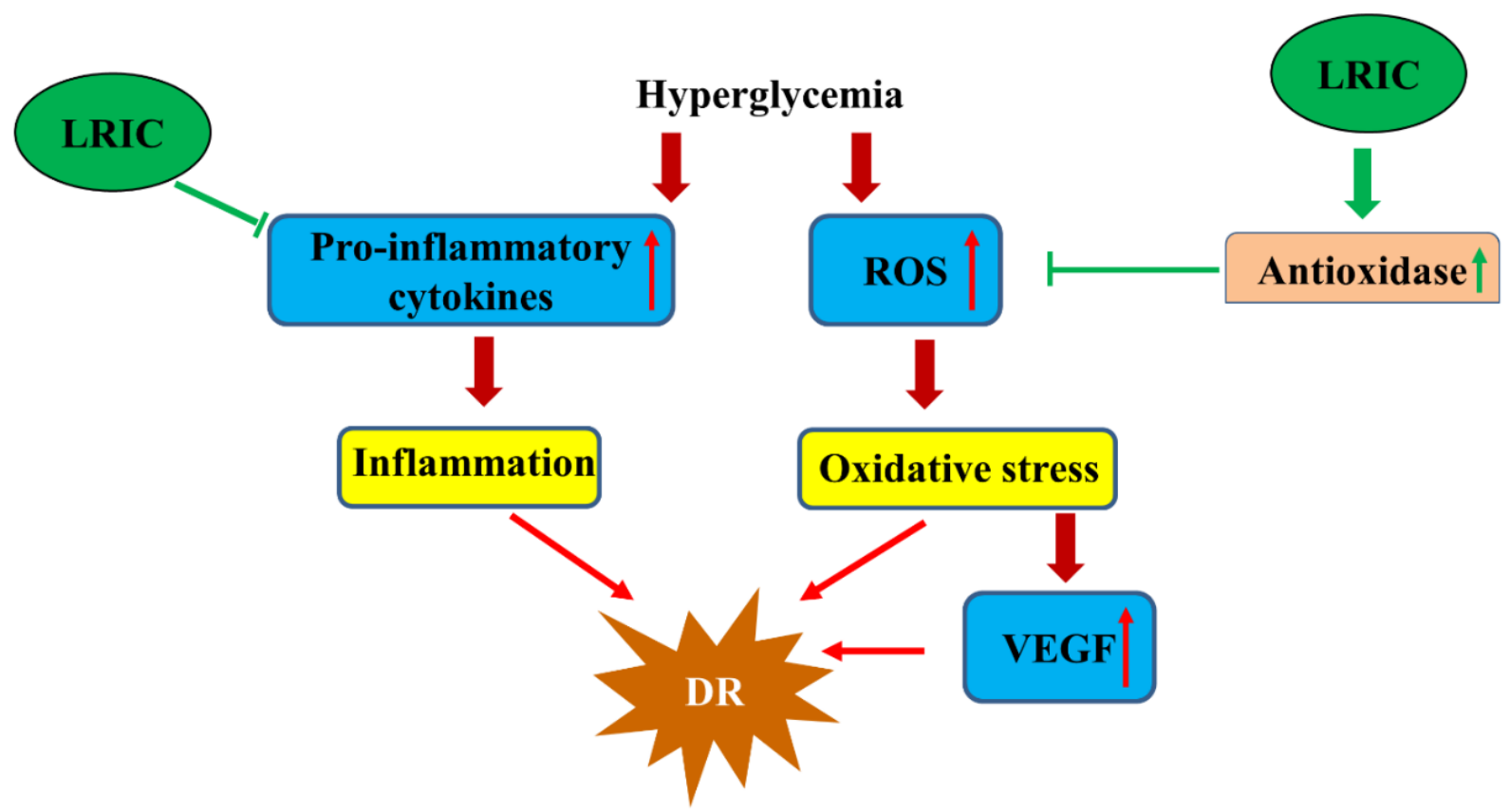

Figure 8. Hypothesis regarding the mechanism of LRIC's protective effects against diabetic retinopathy.

\section{Conclusion}

LRIC ameliorates ganglion cell loss and ameliorated retinal Müller cell activation in STZ-induced diabetic rats. LRIC enhances the activities of SOD and GSH, increases GSH/GSSG ratio and inhibits the production of ROS, IL6 , and VEGF. These results suggest that the antioxidative and anti-inflammatory activities of LRIC may be important mechanisms involved in the protective effect of LRIC on DR in STZ-induced diabetic rats.

\section{Acknowledgements}

This work was supported by the National Key R\&D Program of China (2017YFC1308402), Natural Science Foundation of China (grant number 81573867), and Beijing Municipal Administration of Hospitals Clinical Medicine Development of Special Funding Support (ZYLX201706). 


\section{References}

[1] Fong DS, Aiello L, Gardner TW, King GL, Blankenship G, Cavallerano JD, et al. (2004). Retinopathy in diabetes. Diabetes Care, 27 Suppl 1: S84-87

[2] Jindal V (2015). Neurodegeneration as a primary change and role of neuroprotection in diabetic retinopathy. Mol Neurobiol, 51: 878-884

[3] Hendrick AM, Gibson MV, Kulshreshtha A (2015). Diabetic Retinopathy. Prim Care, 42: 451-464

[4] SooHoo JR, Seibold LK, Kahook MY (2014). The link between intravitreal antivascular endothelial growth factor injections and glaucoma. Curr Opin Ophthalmol, 25: 127-133

[5] Yang Y, Ren C, Zhang Y, Wu X (2017). Ginseng: An Nonnegligible Natural Remedy for Healthy Aging. Aging Dis, 8: 708-720

[6] Luo X, Wu J, Jing S, Yan LJ (2016). Hyperglycemic Stress and Carbon Stress in Diabetic Glucotoxicity. Aging Dis, 7: 90-110

[7] Murugeswari P, Shukla D, Rajendran A, Kim R, Namperumalsamy P, Muthukkaruppan V (2008). Proinflammatory cytokines and angiogenic and antiangiogenic factors in vitreous of patients with proliferative diabetic retinopathy and eales' disease. Retina, 28: 817-824

[8] Masuda T, Shimazawa M, Hara H (2017). Retinal Diseases Associated with Oxidative Stress and the Effects of a Free Radical Scavenger (Edaravone). Oxid Med Cell Longev, 2017: 9208489

[9] Augustin AJ, Keller A, Koch F, Jurklies B, Dick B (2001). [Effect of retinal coagulation status on oxidative metabolite and VEGF in 208 patients with proliferative diabetic retinopathy]. Klin Monbl Augenheilkd, 218: 8994

[10] Heusch G (2013). Cardioprotection: chances and challenges of its translation to the clinic. Lancet, 381: 166-175

[11] Li X, Ren C, Li S, Han R, Gao J, Huang Q, et al. (2017). Limb Remote Ischemic Conditioning Promotes Myelination by Upregulating PTEN/Akt/mTOR Signaling Activities after Chronic Cerebral Hypoperfusion. Aging Dis, 8: 392-401

[12] Fernandez DC, Sande PH, Chianelli MS, Aldana Marcos HJ, Rosenstein RE (2011). Induction of ischemic tolerance protects the retina from diabetic retinopathy. Am J Pathol, 178: 2264-2274

[13] Salido EM, Dorfman D, Bordone M, Chianelli MS, Sarmiento MI, Aranda M, et al. (2013). Ischemic conditioning protects the rat retina in an experimental model of early type 2 diabetes. Exp Neurol, 240: 1-8

[14] Meng R, Asmaro K, Meng L, Liu Y, Ma C, Xi C, et al. (2012). Upper limb ischemic preconditioning prevents recurrent stroke in intracranial arterial stenosis. Neurology, 79: 1853-1861

[15] Brandli A (2015). Remote Limb Ischemic Preconditioning: A Neuroprotective Technique in Rodents. J Vis Exp,
[16] Meng R, Ding Y, Asmaro K, Brogan D, Meng L, Sui M, et al. (2015). Ischemic Conditioning Is Safe and Effective for Octo- and Nonagenarians in Stroke Prevention and Treatment. Neurotherapeutics, 12: 667677

[17] Zhang X, Jizhang Y, Xu X, Kwiecien TD, Li N, Zhang $Y$, et al. (2014). Protective effects of remote ischemic conditioning against ischemia/reperfusion-induced retinal injury in rats. Vis Neurosci, 31: 245-252

[18] Ren C, Gao M, Dornbos D, 3rd, Ding Y, Zeng X, Luo Y, et al. (2011). Remote ischemic post-conditioning reduced brain damage in experimental ischemia/reperfusion injury. Neurol Res, 33: 514-519

[19] Liu ZJ, Chen C, Li XR, Ran YY, Xu T, Zhang Y, et al. (2016). Remote Ischemic Preconditioning-Mediated Neuroprotection against Stroke is Associated with Significant Alterations in Peripheral Immune Responses. CNS Neurosci Ther, 22: 43-52

[20] Rolova T, Dhungana H, Korhonen P, Valonen P, Kolosowska N, Konttinen H, et al. (2016). Deletion of Nuclear Factor kappa B p50 Subunit Decreases Inflammatory Response and Mildly Protects Neurons from Transient Forebrain Ischemia-induced Damage. Aging Dis, 7: 450-465

[21] Geng X, Fu P, Ji X, Peng C, Fredrickson V, Sy C, et al. (2013). Synergetic neuroprotection of normobaric oxygenation and ethanol in ischemic stroke through improved oxidative mechanism. Stroke, 44: 1418-1425

[22] Urzua U, Chacon C, Lizama L, Sarmiento S, Villalobos P, Kroxato B, et al. (2017). Parity History Determines a Systemic Inflammatory Response to Spread of Ovarian Cancer in Naturally Aged Mice. Aging Dis, 8: 546-557

[23] Zheng H, Wu J, Jin Z, Yan LJ (2017). Potential Biochemical Mechanisms of Lung Injury in Diabetes. Aging Dis, 8: 7-16

[24] Nadal-Nicolas FM, Jimenez-Lopez M, Salinas-Navarro M, Sobrado-Calvo P, Alburquerque-Bejar JJ, Vidal-Sanz M, et al. (2012). Whole number, distribution and coexpression of brn3 transcription factors in retinal ganglion cells of adult albino and pigmented rats. PLoS One, 7: e49830

[25] Nadal-Nicolas FM, Jimenez-Lopez M, Sobrado-Calvo P, Nieto-Lopez L, Canovas-Martinez I, Salinas-Navarro M, et al. (2009). Brn3a as a marker of retinal ganglion cells: qualitative and quantitative time course studies in naive and optic nerve-injured retinas. Invest Ophthalmol Vis Sci, 50: 3860-3868

[26] Scott TM, Foote J, Peat B, Galway G (1986). Vascular and neural changes in the rat optic nerve following induction of diabetes with streptozotocin. J Anat, 144: $145-152$

[27] Lopes de Faria JM, Russ H, Costa VP (2002). Retinal nerve fibre layer loss in patients with type 1 diabetes mellitus without retinopathy. Br J Ophthalmol, 86: 725728

[28] Rungger-Brandle E, Dosso AA, Leuenberger PM (2000). Glial reactivity, an early feature of diabetic retinopathy. Invest Ophthalmol Vis Sci, 41: 1971-1980

[29] Dehdashtian E, Mehrzadi S, Yousefi B, Hosseinzadeh A, Reiter RJ, Safa M, et al. (2018). Diabetic retinopathy 
pathogenesis and the ameliorating effects of melatonin; involvement of autophagy, inflammation and oxidative stress. Life Sci, 193: 20-33

[30] Ren C, Li S, Liu K, Rajah G, Han R, Liu Y, et al. (2017). Enhanced oxidative stress response and neuroprotection of combined limb remote ischemic conditioning and atorvastatin after transient ischemic stroke in rats. Brain Circulation, 3: 204-212

[31] Joussen AM, Poulaki V, Le ML, Koizumi K, Esser C, Janicki H, et al. (2004). A central role for inflammation in the pathogenesis of diabetic retinopathy. FASEB J, 18: $1450-1452$

[32] Mocan MC, Kadayifcilar S, Eldem B (2006). Elevated intravitreal interleukin-6 levels in patients with proliferative diabetic retinopathy. Can J Ophthalmol, 41: 747-752

[33] Gustavsson C, Agardh CD, Hagert P, Agardh E (2008). Inflammatory markers in nondiabetic and diabetic rat retinas exposed to ischemia followed by reperfusion. Retina, 28: 645-652

[34] Costagliola C, Daniele A, dell'Omo R, Romano MR, Aceto F, Agnifili L, et al. (2013). Aqueous humor levels of vascular endothelial growth factor and adiponectin in patients with type 2 diabetes before and after intravitreal bevacizumab injection. Exp Eye Res, 110: 50-54

[35] Dongare S, Gupta SK, Mathur R, Saxena R, Mathur S, Agarwal R, et al. (2016). Zingiber officinale attenuates retinal microvascular changes in diabetic rats via antiinflammatory and antiangiogenic mechanisms. Mol Vis, 22: 599-609

[36] Zhao G, Cheng XW, Piao L, Hu L, Lei Y, Yang G, et al. (2017). The Soluble VEGF Receptor sFlt-1 Contributes to Impaired Neovascularization in Aged Mice. Aging Dis, 8: 287-300

[37] Kastelan S, Tomic M, Gverovic Antunica A, Salopek Rabatic J, Ljubic S (2013). Inflammation and pharmacological treatment in diabetic retinopathy. Mediators Inflamm, 2013: 213130

[38] Joussen AM, Poulaki V, Qin W, Kirchhof B, Mitsiades N, Wiegand SJ, et al. (2002). Retinal vascular endothelial growth factor induces intercellular adhesion molecule-1 and endothelial nitric oxide synthase expression and initiates early diabetic retinal leukocyte adhesion in vivo. Am J Pathol, 160: 501-509

[39] Ueno K, Samura M, Nakamura T, Tanaka Y, Takeuchi Y, Kawamura D, et al. (2016). Increased plasma VEGF levels following ischemic preconditioning are associated with downregulation of miRNA-762 and miR-3072-5p. Sci Rep, 6: 36758

[40] Ara J, Fekete S, Frank M, Golden JA, Pleasure D, Valencia I (2011). Hypoxic-preconditioning induces neuroprotection against hypoxia-ischemia in newborn piglet brain. Neurobiol Dis, 43: 473-485

[41] Hausenloy DJ, Yellon DM (2011). The therapeutic potential of ischemic conditioning: an update. Nat Rev Cardiol, 8: 619-629

[42] Fernandez DC, Pasquini LA, Dorfman D, Aldana Marcos HJ, Rosenstein RE (2012). Early distal axonopathy of the visual pathway in experimental diabetes. Am J Pathol, 180: 303-313
[43] Hess DC, Blauenfeldt RA, Andersen G, Hougaard KD, Hoda MN, Ding Y, et al. (2015). Remote ischaemic conditioning-a new paradigm of self-protection in the brain. Nat Rev Neurol, 11: 698-710 